\title{
Design of a Novel Nonlinear Observer to Estimate Sideslip Angle and Tire Forces for Distributed Electric Vehicle
}

\author{
Chuanxue Song, Feng Xiao, Shixin Song, Shaokun Li, and Jianhua Li \\ State Key Laboratory of Automotive Simulation and Control, Jilin University, Changchun 130022, China \\ Correspondence should be addressed to Jianhua Li; ljh_lotus@126.com
}

Received 10 May 2015; Revised 21 September 2015; Accepted 28 September 2015

Academic Editor: Gisella Tomasini

Copyright (C) 2015 Chuanxue Song et al. This is an open access article distributed under the Creative Commons Attribution License, which permits unrestricted use, distribution, and reproduction in any medium, provided the original work is properly cited.

\begin{abstract}
For four-wheel independently driven (4WD) distributed electric vehicle (DEV), vehicle dynamics control systems such as direct yaw moment control (DYC) can be easily achieved. Accurate estimation of vehicle state variables and uncertain parameters can improve the robustness of vehicle dynamics control system. Various sensors are generally equipped to the acquisition of the vehicle dynamics. For both technical and economic reasons, some fundamental vehicle parameters, such as the sideslip angle and tire-road forces, can hardly be obtained through sensors directly. Therefore, this paper presented a state observer to estimate these variables based on Unscented Kalman Filter (UKF). To improve the accuracy of UKF, measurement noise covariance is also self-adaptive regulated. In addition, a nonlinear dynamics tire model is utilized to improve the accuracy of tire lateral force estimation. The simulation and experiment results show that the proposed observer can provide the precision values of the vehicle state.
\end{abstract}

\section{Introduction}

With the development of science and technology, drive system of electric vehicle is also undergoing continuous progress and innovation and individual-wheel drive electric vehicle based on in-wheel motors represents one of the groundbreaking drive structures [1]. Through independent control of the driving torque of each wheel, in-wheel motor driven electric vehicle can achieve direct DYC control. Compared with active DYC system, the DYC of vehicle with in-wheel motors, featuring quick response and precise control, is easier to be achieved [2,3]. With regard to the DYC of in-wheel motor driven vehicles, while yaw rate and sideslip angle are the usual choices for controlled plants, driving torques of the four motors can be taken as the control inputs. So, there are four inputs in the control system. Therefore, the DYC system of in-wheel motor can be seen as a kind of excessive freedom control system which can appropriately assign the torque of each motor, making use of the tire-road force of each tire to its fullest potential and avoiding wheel slip and locking [4].

The accurate and real-time measurement of the statespace is the key for DYC [5]. With the measurement, the ECU can compute the desired dynamics values and detect the stable status of the vehicle. The state-space contains yaw rate and sideslip angle. Yaw rate can be measured by sensors easily. However, it is always not easy to obtain the sideslip angle directly with high accuracy and low expense. Recently, sideslip angle is mainly acquired through observer. The nonlinear factors of the vehicle model and the variation of the vehicle parameters are essential in the sideslip angle estimation. During the cornering, the nonlinear mechanics characteristics of the tires may generate the drastic fluctuations. Algorithms such as sliding mode observer, robustness observer, and Kalman filter observer are adopted in the estimation of the vehicle state [6-8]. EKF (Extended Kalman Filter) is extensively used, especially in the estimation of the tire model $[9,10]$. In the practical application of EKF, the approximate linearization of nonlinear system is performed through Taylor expansion, which can introduce truncation error and make the estimation of parameter hardly achieve high precision. In addition, to calculate Jacobian matrix of the EKF is very complicated. In order to overcome the flaws of EKF, the Unscented Kalman Filter (UKF) algorithm was proposed by Julier and Uhlmann from the University of Oxford [11]. UKF is a novel Kalman Filter based on deterministic sampling approach, and when propagated through the true 


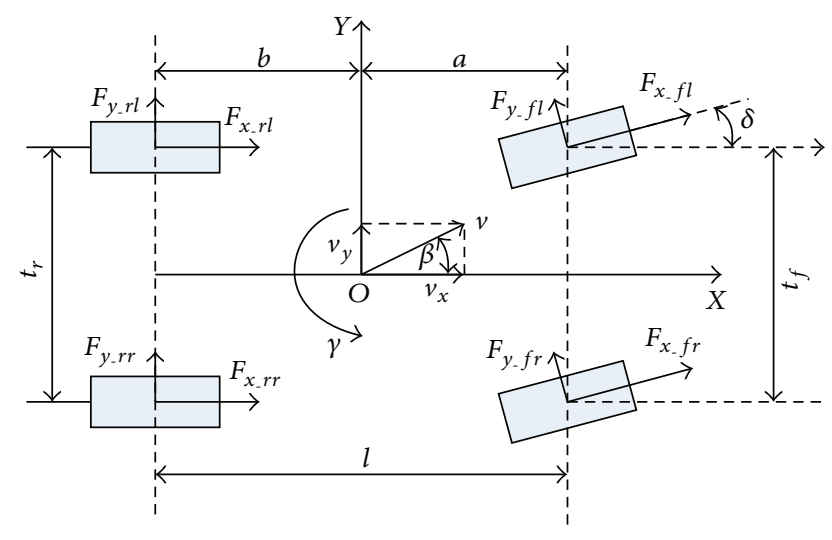

FIGURE 1: Vehicle model.

nonlinear system, these sample points completely capture the true mean and covariance of the Gaussian random variable (GRV) to the 3rd order (Taylor expansion) for any nonlinearity $[12,13]$. In contrast, the EKF only achieves first-order accuracy. So, the nonlinear performance of UKF is superior to EKF remarkably. Doumiati et al. applied UKF to estimate the tire lateral forces and compared the results to those acquired in the EKF observer $[14,15]$. For the 4WD distributed electric vehicle, the distributed driving motor is not only the rapid response of the control unit but also the vehicle information units. Therefore, the distributed electric drive vehicle can provide accurate driving torque and rotational speed, and the information of the sensing range of the vehicle has a greater extension than the traditional vehicle which can make a breakthrough in traditional vehicle dynamics control system using the vehicle parameter estimation based on inertial sensor and reference wheel speed. Provided that a more suitable vehicle model is to be built, the kinematic and dynamic information can be reasonably integrated, which will improve the accuracy of state estimation greatly [16]. Based on the characteristic that distributed electric vehicle (DEV) has multi-information sources, this paper presented an observer to estimate the vehicle state based on Unscented Kalman Filter (UKF). To improve the accuracy of UKF, measurement noise covariance is also self-adaptive regulated. In addition, a nonlinear dynamics tire model is utilized to improve the accuracy of tire lateral force estimation. The performance of the proposed observer is demonstrated through simulation and experiment.

\section{Design of Dynamics Model}

2.1. Vehicle Model. In Figure 1, the 3-DOF vehicle model to be used for the estimator design is illustrated. This model is capable of representing the essential dynamics properties of a road vehicle, incorporating the longitudinal, lateral, and yaw dynamics $[17,18]$.

The differential equations with respect to the longitudinal, lateral, and yaw dynamics are expressed as (1)-(3), respectively. Consider

$$
a_{x}=\dot{v}_{x}-r v_{y}
$$

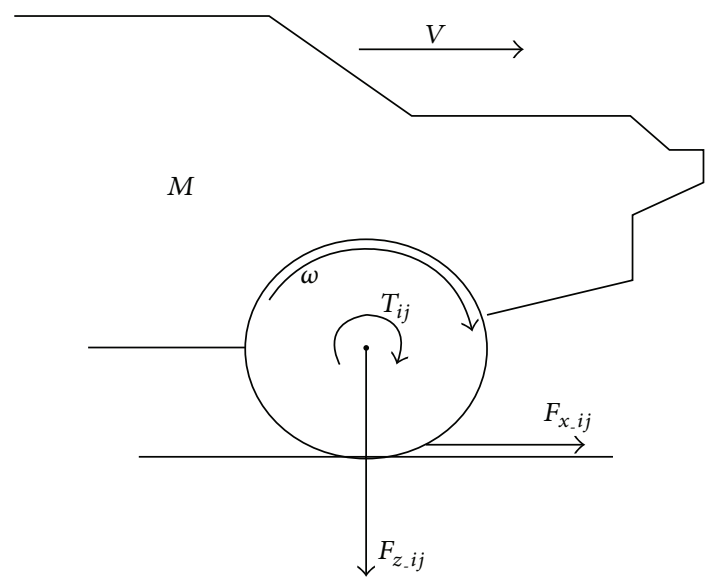

FIGURE 2: Quarter vehicle model.

$$
\begin{aligned}
& a_{y}=\dot{v}_{y}-r v_{x}, \\
& I_{z} \dot{\gamma}=M_{z},
\end{aligned}
$$

where $v_{x}$ is longitudinal velocity of CG $(\mathrm{m} / \mathrm{s}), v_{y}$ is lateral velocity of CG $(\mathrm{m} / \mathrm{s}), \gamma$ is yaw rate $(\mathrm{rad} / \mathrm{s}), a_{x}$ is longitudinal acceleration of CG $\left(\mathrm{m} / \mathrm{s}^{2}\right), a_{y}$ is lateral acceleration of CG $\left(\mathrm{m} / \mathrm{s}^{2}\right), a, b$ are distance between the CG and the front axle and rear axle, respectively $(\mathrm{m}), t_{f}, t_{r}$ are front and rear wheel track, respectively, $\delta$ is front wheel steering angle, $F_{x i j}$ is longitudinal force $(\mathrm{N})$, and $F_{y i j}$ is lateral force $(\mathrm{N}), i j=$ $f l, f r, r l, r r$.

Note that the first subscript in the symbols for the tire force is used to denote front or rear wheel and the second subscript is used to denote left or right wheel.

$$
\begin{aligned}
& M_{z} \text { is the direct yaw moment: } \\
& \begin{array}{l}
M_{z}=a\left(F_{x_{-} f l} \sin \delta+F_{y_{-} f l} \cos \delta+F_{x_{-} f r} \sin \delta\right. \\
\left.\quad+F_{y_{-} f r} \cos \delta\right)-\frac{t_{f}}{2}\left(F_{x_{-} f r} \cos \delta-F_{y_{-} f r} \sin \delta\right. \\
\left.\quad-F_{x_{-} f l} \cos \delta+F_{y_{-} f l} \sin \delta\right)-b\left(F_{y_{-} r l}+F_{y_{-} r r}\right) \\
+\frac{t_{r}}{2}\left(F_{x_{-} r r}-F_{x_{-} r l}\right) .
\end{array}
\end{aligned}
$$

Sideslip angle of CG can be defined as

$$
\beta=\arctan \frac{v_{y}}{v_{x}} .
$$

2.2. Wheel Dynamics. Force analysis of the wheel can be shown in Figure 2.

The rotational dynamics of the 4 wheels are given by the following torque balance equation:

$$
\left(F_{x_{-} i j}+F_{z_{-} i j} f_{i j}\right) r_{i j}=T_{i j}-J_{i j} \dot{\omega}_{i j},
$$

where $T_{i j}$ refer to the drive/brake torque transmitted to the corresponding wheel, $J_{i j}$ refers to the rotational inertia, $\omega_{i j}$ 
refers to the wheel velocity of the corresponding wheel, $F_{z i j}$ refers to the vertical force on the corresponding wheel, $f_{i j}$ refers to the rolling resistance coefficient, and $r_{i j}$ refers to the effective rolling radius of the corresponding wheel.

Hence, the longitudinal force can be expressed as

$$
F_{x_{-} i j}=\frac{1}{r}\left(T_{i j}-J_{i j} \dot{\omega}_{i j}\right)-F_{z_{-} i j} f_{i j} .
$$

The vertical force is given by

$$
\begin{aligned}
& F_{z_{-} f l}=M\left(g \frac{a}{2 l}-a_{x} \frac{h_{g}}{2 l}-a_{y} \frac{h_{g} b}{t_{f} l}\right), \\
& F_{z_{-} f r}=M\left(g \frac{b}{2 l}-a_{x} \frac{h_{g}}{2 l}-a_{y} \frac{h_{g} b}{t_{f} l}\right), \\
& F_{z_{-} r l}=M\left(g \frac{a}{2 l}+a_{x} \frac{h_{g}}{2 l}-a_{y} \frac{h_{g} b}{t_{f} l}\right), \\
& F_{z_{-} r r}=M\left(g \frac{b}{2 l}+a_{x} \frac{h_{g}}{2 l}+a_{y} \frac{h_{g} b}{t_{f} l}\right) .
\end{aligned}
$$

2.3. Tire Model. An appropriate tire model needs to be selected to estimate the lateral force estimation in this paper. The quality of the observer largely depends on the accuracy of the tire model, and the selected model must be precise. The model of the tire-road contact forces is complex, which is affected by vertical load, tire pressure, friction coefficient, vehicle speed, and so forth. Based on the physical characteristic or the empirical formulations derived from experimental data, a few tire models such as Pacejka and Dugoff models can be found in the literature $[19,20]$. And Pacejkas "magic formula" is most commonly used in these models, which can predict the tire behavior precisely. However a lot of parameters are needed in Pacejka model, especially some parameters that are not easy to obtained. By reason of realtime calculation, Dugoff tire model is utilized for estimating the tire lateral forces.

Neglecting longitudinal forces, the lateral tire force is given by

$$
F_{y_{-} i j}=-C_{i j} \tan \alpha_{i j} f(\lambda),
$$

where the cornering stiffness of each tire is given by $C_{i j}$ and $\alpha_{i j}$ is the slip angles at the front and rear tires, which is expressed as

$$
\begin{aligned}
& \alpha_{f l, f r}=-\left(\delta-\arctan \left(\frac{v_{y}+a \gamma}{v_{x} \mp\left(t_{f} / 2\right) \gamma}\right)\right), \\
& \alpha_{r l, r r}=\arctan \left(\frac{v_{y}-b \gamma}{v_{x} \mp\left(t_{r} / 2\right) \gamma}\right) .
\end{aligned}
$$

$f(\lambda)$ is given by

$$
f(\lambda)= \begin{cases}(2-\lambda) \lambda, & \text { if } \lambda<1 \\ 1, & \text { if } \lambda \geq 1,\end{cases}
$$

where $\lambda=\mu F_{z_{-i j}} / 2 C_{i j}\left|\tan \alpha_{i, j}\right|$ and $\mu$ is the lateral friction coefficient.

The original Dugoff tire model has a constant stiffness in respect to weight transfer. However the tire cornering stiffness depends on the tire load and the effect of the load transfer actually [21]. Cornering stiffness can be represented by a second-order polynomial. The tire cornering stiffness can be expressed as

$$
C_{y_{-} i j}\left(F_{z}\right)=\left(m F_{z_{-} i j}-n F_{z i j}^{2}\right),
$$

where $m$ and $n$ are the first- and second-order coefficients in the polynomial, respectively. The modified Dugoff tire model uses $C_{y i j}$ instead of $C_{i j}$.

The tire cornering stiffness changes with transfer of the vertical load, which can modify the Dugoff tire model.

In transient conditions, there is a time lag effect for the lateral forces when the slip angle of tire changes. A relaxation length $\tau_{i j}$ can be adopted to formulate the transient behavior of tires [22]. The relaxation length is the distance covered by the tire while the tire force is kicking in, which depends on the lateral elasticity. The relaxation length is not a constant and varies with the change of slip angle, displaying a nonlinear characteristic. The dynamics lateral forces can be given as follows:

$$
\dot{F}_{y_{-} i j}=\frac{v}{\tau_{i j}}\left(-F_{y_{-} i j}+\overline{F_{y_{-} i j}}\right) \text {, }
$$

where $\overline{F_{y_{i}}}$ is the lateral tire force from Dugoff model in quasistatic condition. Equation (13) is used to calculate the lateral tire force when velocity of vehicle changes.

\section{Observer Design}

State estimation model of nonlinear system can be described with state-space equation:

$$
\begin{aligned}
& \dot{x}(t)=f(x(t), u(t))+w(t), \\
& y(t)=h(x(t), u(t))+v(t) .
\end{aligned}
$$

State variables that need to be observed include longitudinal velocity, side velocity, yaw rate, and lateral force of each wheel. Definition of state variable is

$$
\begin{aligned}
x & =\left[\begin{array}{lllllll}
x_{1} & x_{2} & x_{3} & x_{4} & x_{5} & x_{6} & x_{7}
\end{array}\right]^{T} \\
& =\left[\begin{array}{llllllll}
v_{x} & v_{y} & \gamma & F_{x_{-} f l} & F_{x_{-} f r} & F_{x_{-} r l} & F_{x_{-} r r}
\end{array}\right]^{T} .
\end{aligned}
$$

Measured variables include longitudinal acceleration, lateral acceleration, and yaw rate. Definition of measured variable $y$ is

$$
y=\left[\begin{array}{lll}
y_{1} & y_{2} & y_{3}
\end{array}\right]^{T}=\left[\begin{array}{lll}
\gamma & a_{x} & a_{y}
\end{array}\right]^{T} .
$$

Longitudinal force can be calculated from the tire's rotating dynamics equation (6). As the precise moment and revolving speed of the drive motors can be obtained, it can be presumed that the longitudinal forces of wheels are known. 
Therefore, the input variables of state equation are defined to be the steering angles of front wheels and the longitudinal force of each wheel:

$$
\begin{aligned}
u & =\left[\begin{array}{lllll}
u_{1} & u_{2} & u_{3} & u_{4} & u_{5}
\end{array}\right]^{T} \\
& =\left[\begin{array}{llllll}
\delta & F_{x_{-} f l} & F_{x_{-} f r} & F_{x_{-} r l} & F_{x_{-} r r}
\end{array}\right]^{T} .
\end{aligned}
$$

$w$ and $v$ are process noise and measurement noise, respectively, which is in compliance with Gaussian distribution.

Hence, state equation for the observer is

$$
\begin{aligned}
\dot{x}_{1} & =\frac{\left(u_{2} \cos \left(u_{1}\right)-x_{4} \sin \left(u_{1}\right)+u_{3} \cos \left(u_{1}\right)-x_{5} \sin \left(u_{1}\right)+u_{4}+u_{5}-F_{w}\right)}{m}+x_{3} x_{2}, \\
\dot{x}_{2} & =\frac{\left(u_{2} \sin \left(u_{1}\right)+x_{4} \cos \left(u_{1}\right)+u_{3} \sin \left(u_{1}\right)+x_{5} \cos \left(u_{1}\right)+x_{6}+x_{7}\right)}{m}+x_{3} x_{1}, \\
\dot{x}_{3} & =\left[a\left(u_{2} \sin \left(u_{1}\right)+x_{4} \cos \left(u_{1}\right)+u_{3} \sin \left(u_{1}\right)-u_{5} \cos \left(u_{1}\right)\right)-\frac{t_{f}}{2}\left(u_{3} \cos \left(u_{1}\right)-x_{5} \sin \left(u_{1}\right)-u_{2} \cos \left(u_{1}\right)+x_{4} \sin \left(u_{1}\right)\right)\right. \\
& \left.-b\left(x_{6}+x_{7}\right)+\frac{t_{r}}{2}\left(u_{5}-u_{4}\right)\right] I_{z}, \\
\dot{x}_{4} & =\frac{v}{\tau_{f l}}\left(-x_{4}+\bar{F}_{y_{-} f l}\left(\alpha_{f l}, F_{z_{-} f l}\right)\right), \\
\dot{x}_{5} & =\frac{v}{\tau_{f r}}\left(-x_{5}+\bar{F}_{y_{-} f r}\left(\alpha_{f r}, F_{z_{-} f r}\right)\right), \\
\dot{x}_{6} & =\frac{v}{\tau_{r l}}\left(-x_{6}+\bar{F}_{y_{-} r l}\left(\alpha_{r l}, F_{z_{-} r l}\right)\right), \\
\dot{x}_{7}= & \frac{v}{\tau_{r r}}\left(-x_{7}+\bar{F}_{y_{-} r r}\left(\alpha_{r r}, F_{z_{-} r r}\right)\right) .
\end{aligned}
$$

The measurement equation is

$$
\begin{aligned}
h_{1}= & \gamma=x_{3}, \\
h_{2}= & a_{x} \\
= & u_{2} \cos \left(u_{1}\right)-x_{4} \sin \left(u_{1}\right)+u_{3} \cos \left(u_{1}\right) \\
& -x_{5} \sin \left(u_{1}\right)+u_{4}+u_{5}-F_{w}, \\
h_{3}= & a_{y} \\
= & u_{2} \sin \left(u_{1}\right)+x_{4} \cos \left(u_{1}\right)+u_{3} \sin \left(u_{1}\right) \\
& +x_{5} \cos \left(u_{1}\right)+x_{6}+x_{7} .
\end{aligned}
$$

State variables are to be estimated with UKF technology.

\section{Design of UKF}

Based on the complete state estimation model, an appropriate estimation method should be chosen. Kalman filter is one of the most effective mathematical tools in state estimation field, but it can only be used in the liner system estimation. EKF is extensively used in the estimation of vehicle state, especially in the identification of tire model. In the practical application of EKF, the approximate linearization of nonlinear system is performed through Taylor expansion, which can introduce truncation error and make EKF perform badly when the system is severely nonlinear. In order to overcome the flaws of EKF, UKF algorithm was proposed by Julier and Uhlmann from the University of Oxford [11]. Compared with the EKF, the ability of processing nonlinearity without linearization is the advantage of the UKF. UKF is essentially a kind of Quasi-Monte Carlo method [23]. UKF refers to the application of sampling method under Kalman filtering framework to approximate nonlinear function for the reason that to approximate the probability density distribution of nonlinear function is easier than to approximate nonlinear function. The core idea of UKF is UT transformation which uses deterministic variables to approximate a Gaussian distribution. It means choosing several spots from the original state distribution according to certain rules and the average value and covariance of these spots equal the average value and covariance of the original state distribution. When these spots are put into the nonlinear function, the value point set of the corresponding nonlinear function is obtained. From this point set, we can get the transformed average value and covariance. More details are available in [24]. The procedure for estimating the state of in-wheel motor driven vehicle with $\mathrm{UKF}$ is as follows.

(1) Discretization of State Equation. After discretization of state equation (14), we get the following discrete equation of state:

$$
\begin{gathered}
x_{k+1}=f\left(x_{k}, u_{k}\right)+w_{k}, \\
y_{k}=h\left(x_{k}, u_{k}\right)+v_{k},
\end{gathered}
$$


where $x_{k}$ is the state vector at time $k, u_{k}$ is input vector, $y_{k}$ is output vector, covariance of process noise $w_{k}$ is $Q$, covariance of measurement noise $v_{k}$ is $R$, and the cross covariance between $w_{k}$ and $v_{k}$ is $0 . Q$ and $R$ are both symmetric positive definite matrix.

(2) Initialization. System noise and measurement noise are taken into consideration and extended vector is defined as $x_{k}^{a}=\left[\begin{array}{lll}x_{k} & w_{k} & v_{k}\end{array}\right]^{T}$ and the initial conditions conform to

$$
\begin{aligned}
& \bar{x}_{0}=E\left(x_{0}\right), \\
& P_{0}=E\left[\left(x_{0}-\bar{x}_{0}\right)\left(x_{0}-\bar{x}_{0}\right)^{T}\right], \\
& \bar{x}_{0}^{a}=E\left[x^{a}\right]=\left[\begin{array}{lll}
\bar{x}_{0}^{T} & 0 & 0
\end{array}\right]^{T}, \\
& P_{0}^{a}=E\left[\left(x_{0}^{a}-\bar{x}_{0}^{a}\right)\left(x_{0}^{a}-\bar{x}_{0}^{a}\right)^{T}\right] .
\end{aligned}
$$

(3) Sample of Sigma Points. Consider

$$
\begin{aligned}
& \chi_{0, k \mid k}^{a}=\bar{x}_{k \mid k}^{a}, \\
& \chi_{i, k \mid k}^{a}=\bar{x}_{k \mid k}^{a}+\sqrt{\left(n_{a}+\lambda\right) P_{k \mid k}^{a}}, \quad i=1, \ldots, n, \\
& \chi_{i, k \mid k}^{a}=\bar{x}_{k \mid k}^{a}-\sqrt{\left(n_{a}+\lambda\right) P_{k \mid k}^{a}}, \quad i=n+1, \ldots, 2 n .
\end{aligned}
$$

Associated weights of Sigma points are as follows:

$$
\begin{aligned}
& W_{0}^{(m)}=\frac{\lambda}{\left(n_{a}+\lambda\right)}, \\
& W_{0}^{(c)}=\frac{\lambda}{\left(n_{a}+\lambda\right)}+\left(1-\alpha^{2}+\beta_{t}\right), \\
& W_{i}^{(m)}=W_{i}^{(c)}=\frac{1}{2\left(n_{a}+\lambda\right)}, \quad i=1, \ldots, 2 n,
\end{aligned}
$$

where $n_{a}=2 n+n_{v}, \lambda=\alpha^{2}\left(n_{a}+e\right)-n_{a}, n$ stands for number of dimensions of state vector, and $n_{v}$ is the number of dimensions of measurement noise.

The values for variables $\alpha, \beta_{t}$, and $e$ should be appropriately chosen. 0 is the usual value for $e$, which ensures the half positive definitiveness of covariance matrix; $\alpha$ determines the density of Sigma around $\bar{x}_{0}^{a}$ and its usual value range is $10^{-4} \leq \alpha \leq 1$. In this paper, $\alpha=0.005$; when the random variables conform to Gaussian distribution, $\beta_{t}=2$ is the most appropriate.

(4) Calculation of UKF Recursive Filtering. Time update equations are as follows:

$$
\begin{aligned}
& \chi_{i, k+1 \mid k}^{a}=f\left(\chi_{i, k \mid k}^{a}, u_{k}\right), \\
& \bar{x}_{k+1 \mid k}^{a}=\sum_{i=0}^{2 n_{a}} W_{i}^{(m)} \chi_{i, k+1 \mid k}^{a}, \\
& y_{i, k+1 \mid k}^{a}=h\left(\chi_{i, k+1 \mid k}^{a}, u_{k}\right),
\end{aligned}
$$

$$
\begin{aligned}
& \bar{y}_{k+1 \mid k}^{a}=\sum_{i=0}^{2 n_{a}} W_{i}^{(m)} y_{i, k+1 \mid k}^{a}, \\
& P_{k+1 \mid k}^{a} \\
& \quad=\sum_{i=0}^{2 n_{a}} W_{i}^{(c)}\left(\chi_{i, k+1 \mid k}^{a}-\bar{X}_{k+1 \mid k}^{a}\right)\left(\chi_{i, k+1 \mid k}^{a}-\bar{X}_{k+1 \mid k}^{a}\right)^{T} .
\end{aligned}
$$

Measurement update equations are as follows:

$$
\begin{aligned}
& P_{(y, y), k+1 \mid k}^{a} \\
& \quad=\sum_{i=0}^{2 n_{a}} W_{i}^{(c)}\left(y_{k+1 \mid k}^{a}-y_{k+1 \mid k}^{a}\right)\left(y_{k+1 \mid k}^{a}-y_{k+1 \mid k}^{a}\right)^{T}, \\
& P_{(x, y), k+1 \mid k}^{a} \\
& \quad=\sum_{i=0}^{2 n_{a}} W_{i}^{(c)}\left(x_{k+1 \mid k}^{a}-\bar{x}_{k+1 \mid k}^{a}\right)\left(y_{k+1 \mid k}^{a}-\bar{y}_{k+1 \mid k}^{a}\right)^{T}, \\
& K_{k+1}=P_{(x, y), k+1 \mid k}^{a}\left(P_{(y, y), k+1 \mid k}^{a}\right)^{-1}, \\
& P_{k+1 \mid k+1}^{a}=P_{k+1 \mid k}^{a}-K_{k+1} P_{(y, y), k+1 \mid k}^{a} K_{k+1}^{T}, \\
& \bar{x}_{k+1 \mid k+1}^{a}=\bar{x}_{k+1 \mid k}+K_{k+1}\left(y_{k}^{a}-\bar{y}_{k+1 \mid k}^{a}\right) .
\end{aligned}
$$

\section{Adaptive Regulation of Measurement Noise Covariance}

Measured variables are composed of longitudinal acceleration, lateral acceleration, and yaw rate from inertial sensor as shown in (16). Assuming that each measurement signal was independent of each other, the measurement noise covariance matrix $R$ can be simplified to a diagonal matrix

$$
R=\operatorname{diag}\left(\begin{array}{lll}
R_{\gamma} & R_{a_{x}} & R_{a_{y}}
\end{array}\right) .
$$

Although the noise covariance matrix cannot be given accurately, the state information of vehicle is closely related to noise covariance matrix, on the basis of which standards of measurement noise covariance adaptive regulation were given as follows:

(1) When the signals of inertial sensor such as $\gamma, a_{x}$, and $a_{y}$ are small, inertial sensor has low value of Signal Noise Ratio (SNR) and the credibility of the signal reduces. So the measurement noise of inertial sensor $R_{\gamma}, R_{a_{x}}$, and $R_{a_{y}}$ should be increased.

(2) When the value of longitudinal force is large, the longitudinal acceleration of vehicle is large, so the measurement noise of longitudinal acceleration $R_{a_{x}}$ should be reduced.

(3) When the velocity of vehicle is a constant value, the yaw rate would increase with the growth of steering wheel angle. Therefore, the measurement noise corresponding to the value of yaw rate should decrease. 


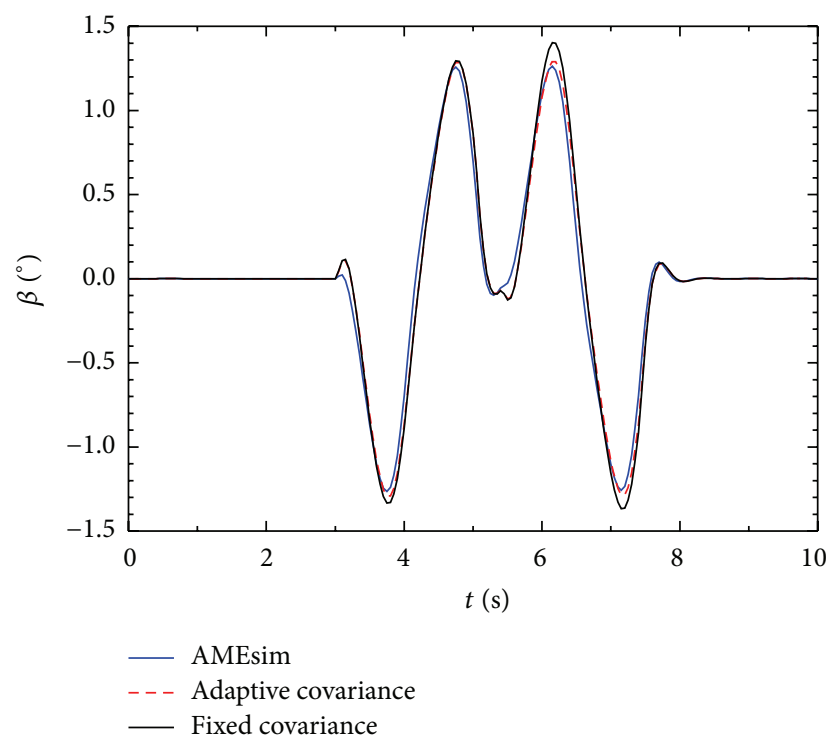

FIgURE 3: Comparison of the estimation values.

(4) The value of the lateral acceleration should become smaller with the decrease of steering wheel angle. On this condition, the noise of lateral acceleration $R_{a_{y}}$ should be increased.

Taking the rules above into consideration, the RISF (Reliability Indexed Sensor Fusion) method is proposed to design the covariance matrix according to the state information of vehicle [25], which is defined by

$$
\begin{aligned}
r_{a_{x}}= & c_{a_{x 1}} \exp \left(-d_{a_{x 1}}\left|a_{x}\right|\right) \\
& +c_{a_{x 2}} \exp \left(-d_{a_{x 2}}\left|\sum_{i j=f l, f r, r l, r r} F_{i j, x}\right|\right) \\
& +c_{a_{x 3}} \exp \left(-d_{a_{x 3}}\left|\sum_{i j=f l, f r, r l, r r} \kappa_{i j}\right|\right), \\
r_{a_{y}=} & c_{a_{y 1}} \exp \left(-d_{a_{y 1}}\left|a_{y}\right|\right)+c_{a_{y 2}} \exp \left(-d_{a_{y 2}}|\delta|\right), \\
r_{\gamma}= & c_{\gamma_{1}} \exp \left(-d_{\gamma_{1}}|\gamma|\right)+c_{\gamma_{2}} \exp \left(-d_{\gamma_{2}}|\delta|\right),
\end{aligned}
$$

where, $c_{m}$ and $d_{m}$ are positive real number and $m \in$ $\left\{a_{x 1}, a_{x 2}, a_{x 3}, a_{y 1}, a_{y 2}, \gamma_{1}, \gamma_{2}\right\}$.

The covariance matrix proposed is applied to the process of UKF, which can improve the observer's capacity of resisting disturbance and enhance the observation precision in multicondition comprehensively.

The main function of the measurement noise covariance adaptive regulation is to improve the estimation accuracy of state parameter, so a simulation test is carried out to compare the estimation values of sideslip angle between the adaptive covariance and fixed covariance. Double lane change test is run at the speed of $80 \mathrm{~km} / \mathrm{h}$.

Figure 3 shows the estimation values of sideslip angle in simulation. In the condition of the measurement noise

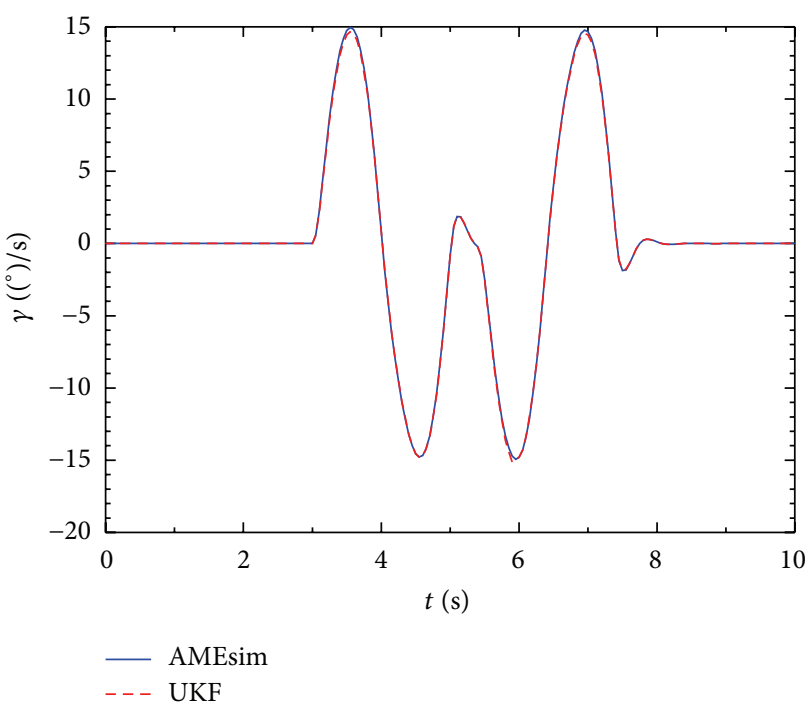

FIGURE 4: Estimation of the yaw rate.

covariance fixed regulation, the estimation precision would reduce when the value of sideslip angle is large. Aggravation of vehicle nonlinear effect is not taken into consideration when the vehicle conducts an intense lateral movement, which can lead to the decrease of the estimation precision. On the contrary, when the measurement noise covariance adaptive regulation is adopted, the credibility of yaw rate and lateral acceleration signals is enhanced to improve the estimation precision.

\section{Simulation Verification}

A simulation test was run in AMEsim so as to verify the observation effect of UKF algorithm. For vehicle dynamics simulation, AMEsim provides a comprehensive solution which includes a special vehicle dynamics application library integrated vehicle module, tire module, pavement module, driver module, and other vehicle subsystem module that high precision dynamic virtual tests can be carried out which improve the user's R \& D efficiency and shorten the development time of new products greatly [26]. The vehicle state from vehicle model can be seen as true value. The yaw rate, lateral acceleration, and longitudinal acceleration of the vehicle can be delivered to the observer as input and finally, the lateral tire forces and sideslip angles estimated by the observer were compared with the true values in the vehicle model for the verification of observation effect of UKF algorithm. Double lane change tests were run on roads with adhesion coefficients of 0.9 and 0.3 , respectively. Step length of simulatlion test was $1 \mathrm{~ms}$.

6.1. Test on Road of High Adhesion. Vehicle speed is $80 \mathrm{~km} / \mathrm{h}$; adhesion coefficient is 0.9 .

Figure 4 shows the estimated results of yaw rate. As yaw rate can be directly obtained from the sensor, the estimation is accurate and the convergence is very quick. Figure 5 shows the estimated results of sideslip angles. It can be seen that the 


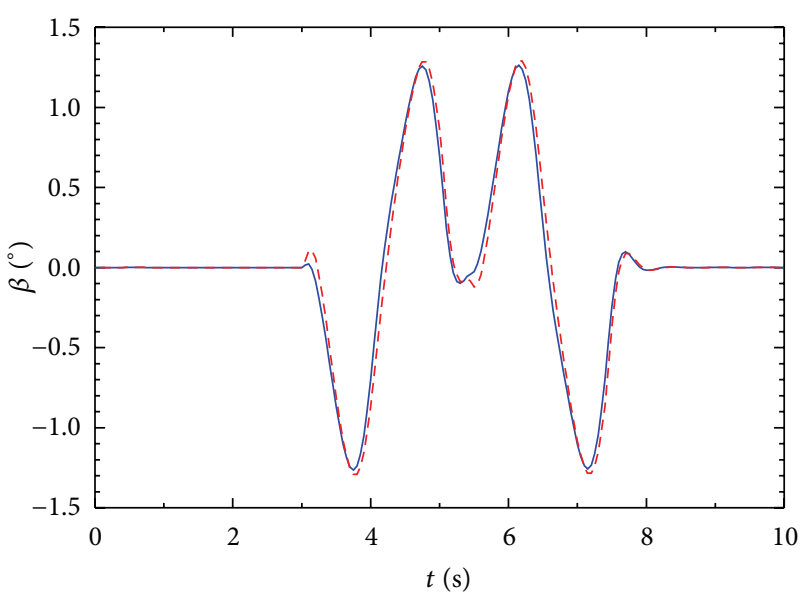

- AMEsim

- - UKF

FIGURE 5: Estimation of the sideslip angle at CG.

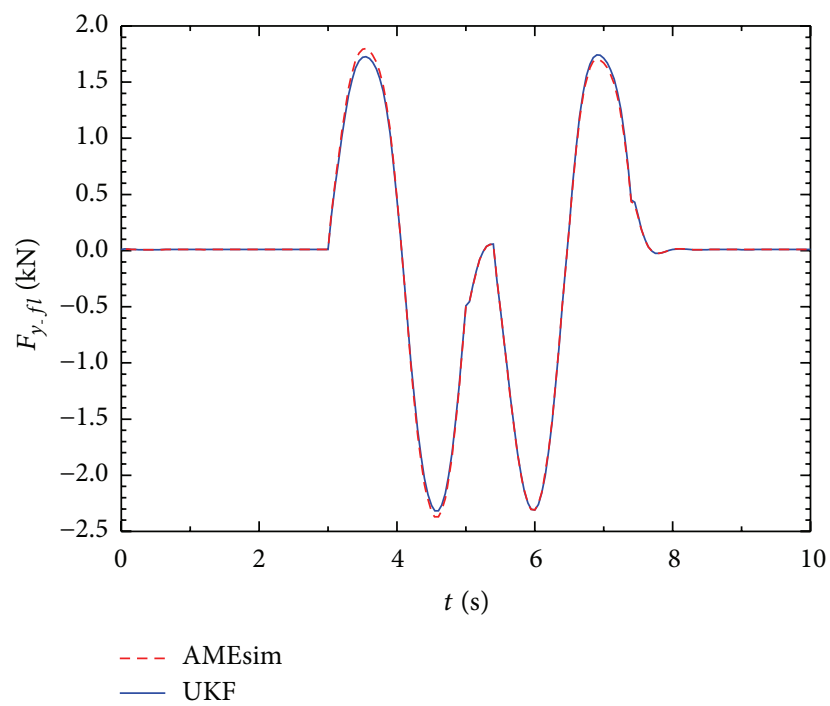

Figure 6: Lateral force of FL tire.

estimated values had very similar tendency with the reference values. When lateral excitation is big, errors of the estimated values would be small. Figures $6-9$ show the estimated values of lateral tire force of the tires. The errors between observed values and reference values are very small and they only increase at wave crest and wave trough.

6.2. Test on Road of Low Adhesion. Vehicle speed is $80 \mathrm{~km} / \mathrm{h}$; adhesion coefficient is 0.3 . When running double lane change on this road, the vehicle made nonlinear movement.

Figure 10 shows that, on the road of low adhesion, estimated values of yaw rate are still highly accurate, which lays a favorable foundation for the accurate estimation of sideslip angles. It can be seen from Figure 11 that observed values of sideslip angles can follow the output values of the vehicle model. It can be noted that within the range of $8-10 \mathrm{~s}$, the sideslip angle reached as high as 19 degrees and the vehicle entered the nonlinear range. Figures $12-15$ show the

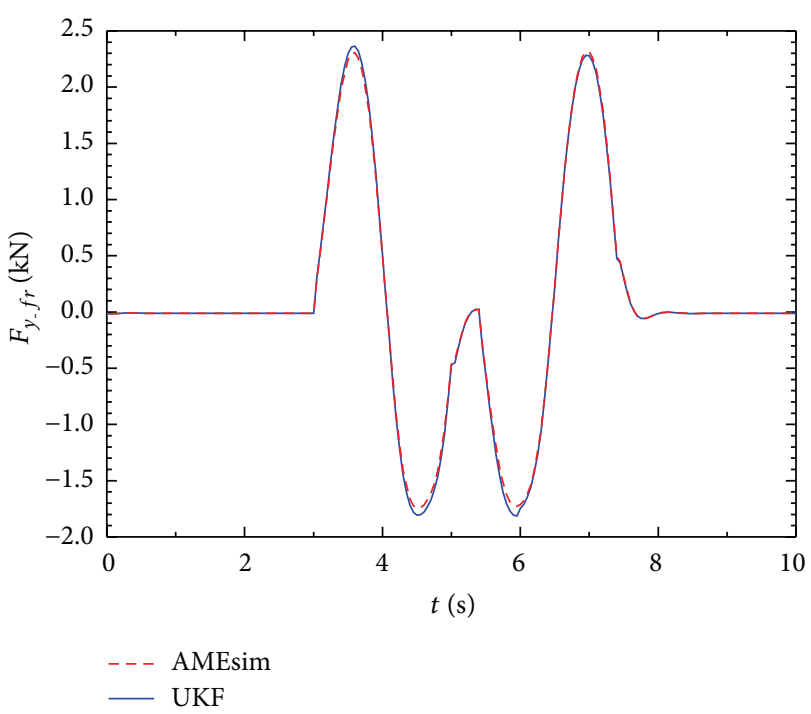

FIGURE 7: Lateral force of FR tire.

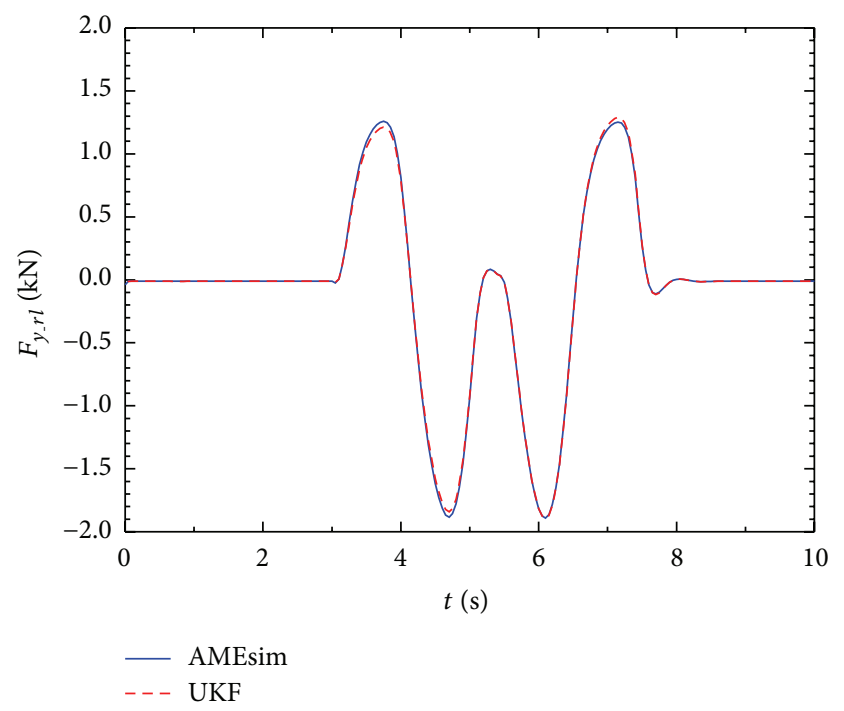

FIGURE 8: Lateral force of RL tire.

observation results of tire lateral force. Errors are larger where sideslip angles are big. At that time, the relation between tire and the ground entered nonlinear range, which changed the trend of force on the tire and affected the observation results.

\section{Experimental Results}

In this section, In order to study experimentally the performance of the vehicle state observer, the road test is carried out on the distributed driving vehicle test platform attached to our research group.

7.1. Experimental Car. The experimental vehicle shown in Figure 16 belongs to State Key Laboratory of ASCL Jilin University. It is a refitted Besturn B50. The rapid control prototyping (RCP) is developed through MATLAB/Simulink, and the observer is embedded in RCP. The dynamic information 


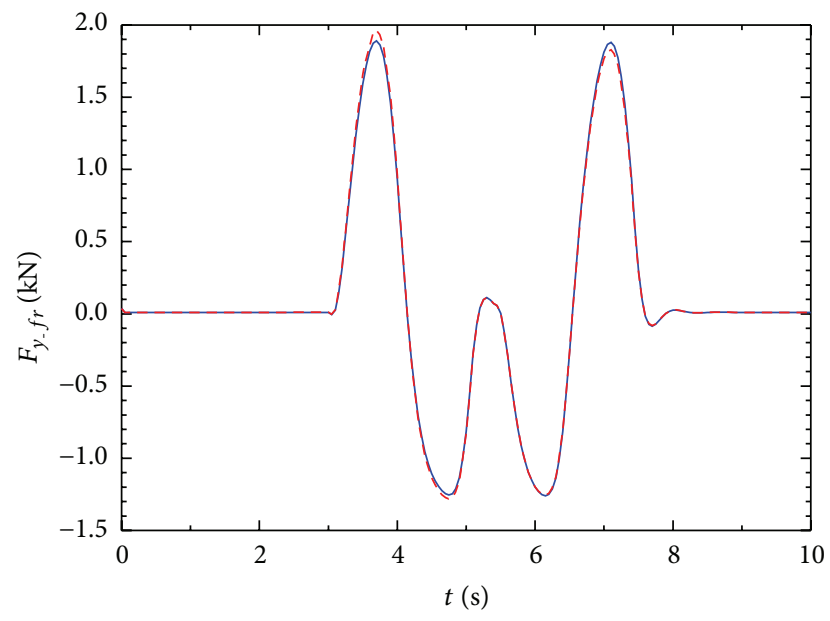

- AMEsim

--- UKF

FIgURE 9: Lateral force of RR tire.

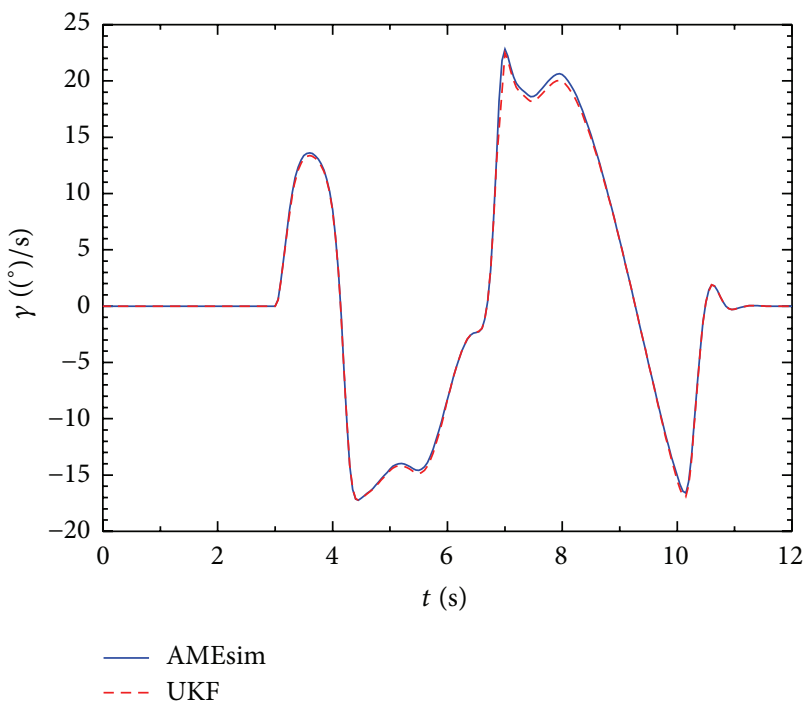

FIGURE 10: Estimation of the yaw rate.

of the vehicle is measured by Oxford Technical Solutions RT3100 shown in Figure 17 and its results are used as the real reference for validating the estimation process. The car is fitted with a measurement device including 8 analog channels and a CAN-Bus interface with 2 nodes. The sampling time is $10 \mathrm{~ms}$.

7.2. Test and Results. A double lane change test is done to validate the observers. Due to the power limit of in-wheel motor, $50 \mathrm{~km} / \mathrm{h}$ is set as the test velocity. The observation results are shown in Figures 18 and 19.

Reported results are relatively good. The observation results are summarized in Table 1. Analysis shows that, under the double lane change condition, the estimation of the sideslip angle and yaw velocity is precise. The force of tires

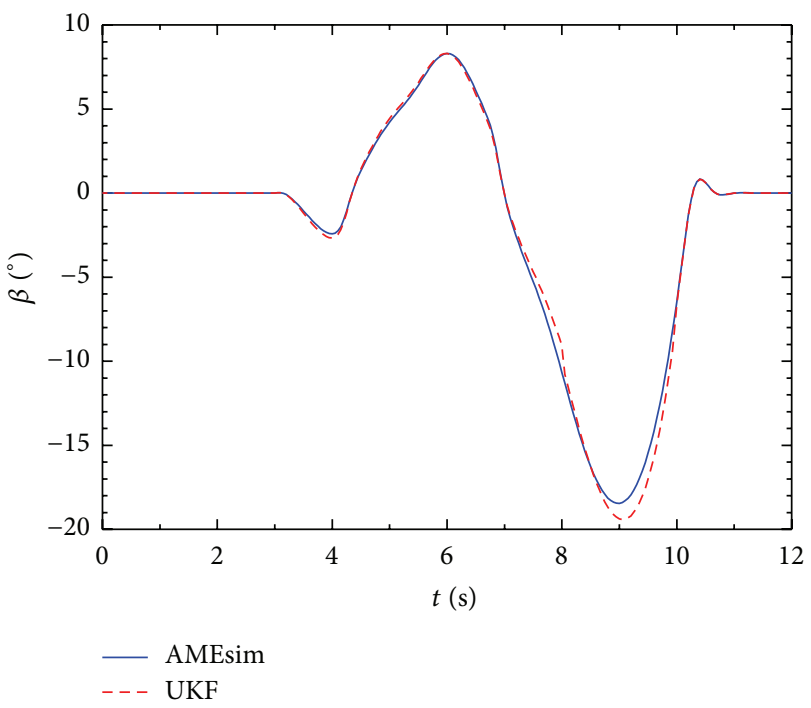

FIgURE 11: Estimation of the sideslip angle at CG.

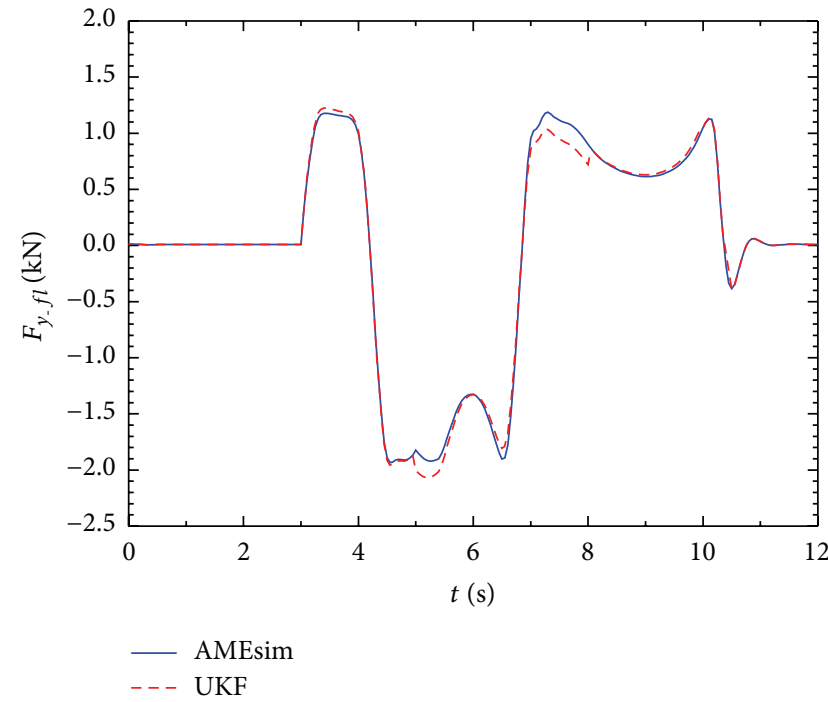

FIGURE 12: Lateral force of FL tire.

TABLE 1

\begin{tabular}{ccccc}
\hline & \multicolumn{2}{c}{ Root mean-squared error } & \multicolumn{2}{c}{ Maximum error } \\
& $\gamma\left[\left(^{\circ}\right) / \mathrm{s}\right]$ & $\beta\left[^{\circ}\right]$ & $\gamma\left[\left(^{\circ}\right) / \mathrm{s}\right]$ & $\beta\left[^{\circ}\right]$ \\
\hline Value & 1.71 & 0.27 & 3.78 & 0.43 \\
\hline
\end{tabular}

is the key parameter for state estimation, and the accuracy of estimation for the sideslip angle and yaw velocity can show that the estimation of tire force is also precise. The performance of observers is satisfactory.

\section{Conclusions}

Accuracy of tire force calculation is key to vehicle state estimation. The modified Dugoff tire model, featuring the consideration of dynamic characteristics, was introduced for 


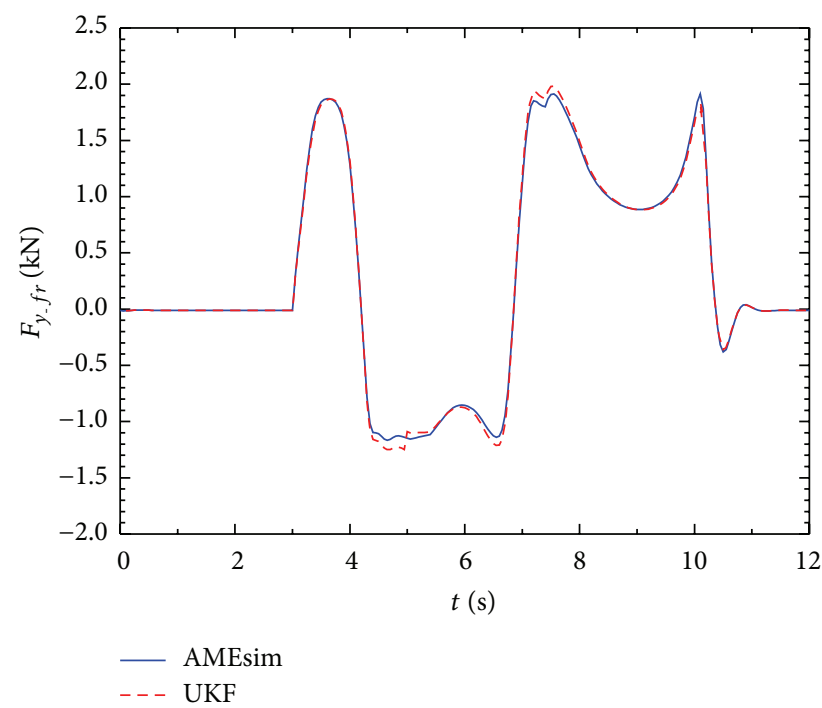

FIGURE 13: Lateral force of FR tire.

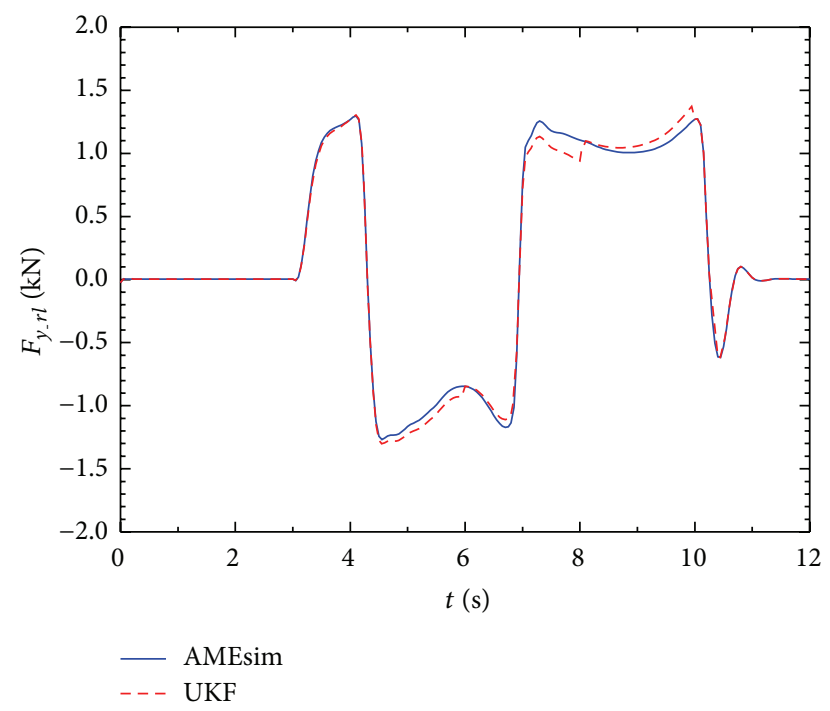

FIGURE 14: Lateral force of RL tire.

revising the observation and calculation of the lateral tire force. Simulation results show that this modified Dugoff tire model can provide effective estimation of the lateral tire force in transient conditions.

The adaptive regulation of the measurement noise covariance can improve the observer's capacity of resisting disturbance and enhance the observation precision in multicondition comprehensively.

This paper makes use of the characteristics of $4 \mathrm{WD}$ distributed electric vehicle, establishes a dynamic model of vehicle, and designs a vehicle state based on the UKF algorithm. The simulation results show that the observer can accurately estimate the vehicle sideslip angle, yaw rate, and lateral tire forces in the field of nonlinear estimation, which is significant for the improvement of vehicle dynamics control. Performances are tested using an experimental car, and experimental results demonstrate the ability of this approach

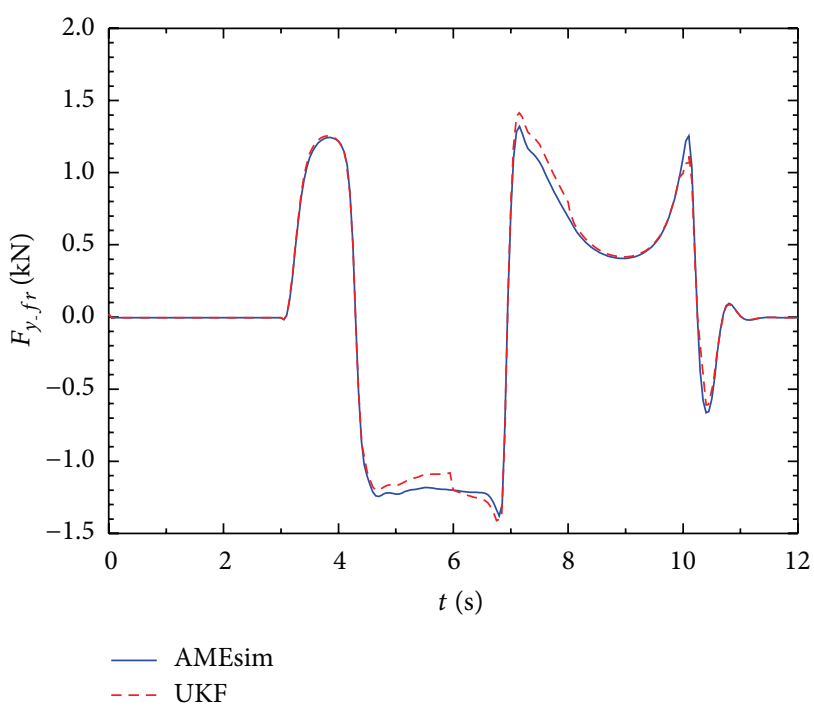

FIGURE 15: Lateral force of RR tire.

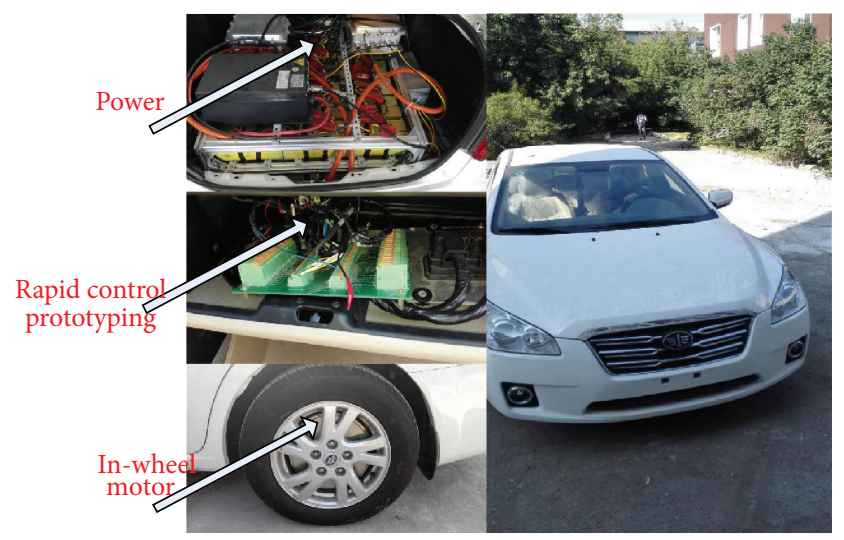

FIGURE 16: Experimental vehicle.

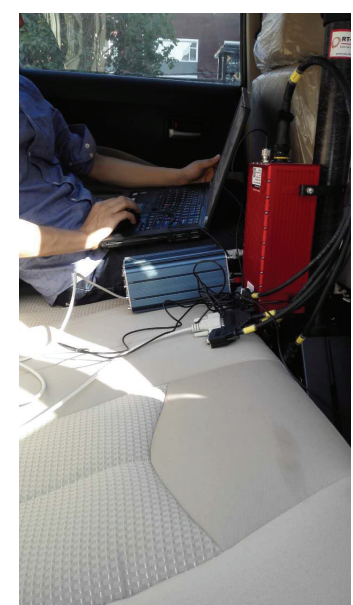

FIgURE 17: Data acquisition system. 


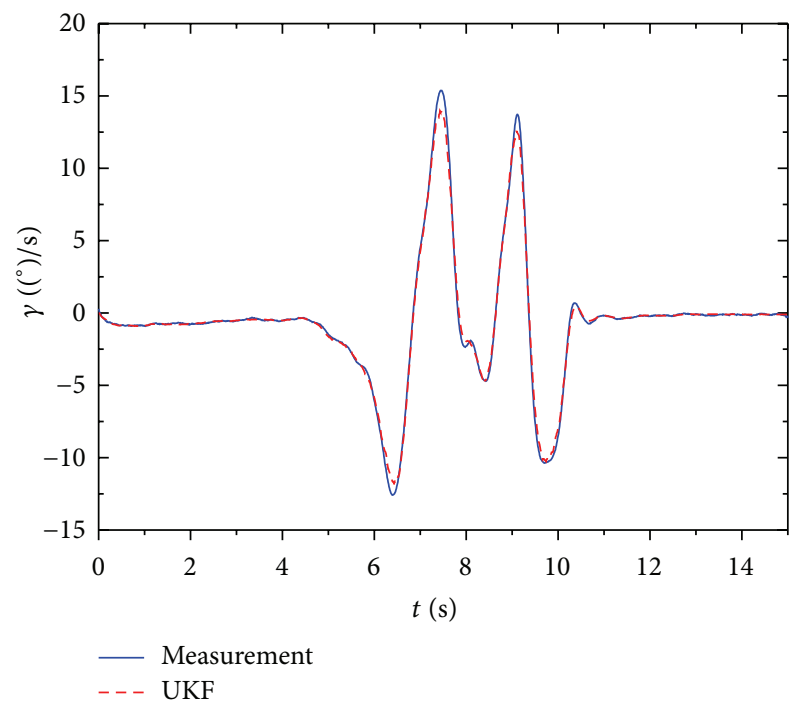

FIGURE 18: Estimation of the yaw rate.

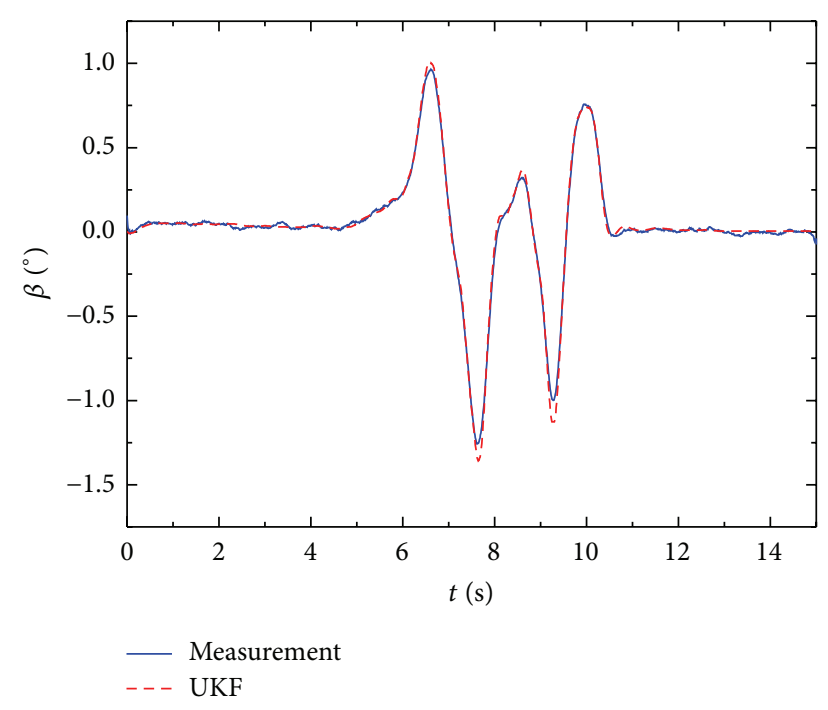

FIGURE 19: Estimation of the sideslip angle.

to provide accurate estimations and show its practical potential as a low-cost solution for calculating lateral tire forces and sideslip angle.

\section{Conflict of Interests}

The authors declare that there is no conflict of interests regarding the publication of this paper.

\section{Acknowledgment}

This work is supported by International Cooperation Projects of Ministry of Science and Technology of China (2010DFB83650).

\section{References}

[1] Y. Hori, "Future vehicle driven by electricity and controlresearch on four-wheel-motored 'UOT Electric March II,' IEEE Transactions on Industrial Electronics, vol. 51, no. 5, pp. 954-962, 2004.

[2] S.-I. Sakai and Y. Hori, "Advanced motion control of electric vehicle with fast minor feedback loops: basic experiments using the 4-wheel motored EV 'UOT Electric March II'” JSAE Review, vol. 22, no. 4, pp. 527-536, 2001.

[3] G. Li, C.-F. Zong, G.-Y. Chen, and W. Hong, "Integrated AFS/DYC control of steering-by-wire 4WID electric vehicle," Journal of South China University of Technology, vol. 40, no. 3, pp. 150-155, 2012.

[4] P. He and Y. Hori, "Resolving actuamr redundancy for 4WD electric vehicle bysequential quadratic optimum method," in Proceedings of the 19th Japan Industry Applications Society Conference, p. 8, Fukui, Japan, 2005.

[5] Z. Yu and X. Gao, "Review of vehicle state estimation problem under driving situation," Journal of Mechanical Engineering, vol. 45 , no. 5, pp. 20-33, 2009.

[6] J. Stéphant, A. Charara, and D. Meizel, "Evaluation of a sliding mode observer for vehicle sideslip angle," Control Engineering Practice, vol. 15, no. 7, pp. 803-812, 2007.

[7] D. W. Pi, N. Chen, J. X. Wang, and B. J. Zhang, "Design and evaluation of sideslip angle observer for vehicle stability control," International Journal of Automotive Technology, vol. 12, no. 3, pp. 391-399, 2011.

[8] C. Ahn, H. Peng, and H. Tseng, "Robust nonlinear observer to estimate road friction coefficient and tire slip angle," in Proceedings of the 10th International Symposium on Advanced Vehicle Control (AVEC '10), Loughborough, UK, August 2010.

[9] T. A. Wenzel, K. J. Burnham, M. V. Blundell, and R. A. Williams, "Dual extended Kalman filter for vehicle state and parameter estimation," Vehicle System Dynamics, vol. 44, no. 2, pp. 153-171, 2006.

[10] J. Kim, "Identification of lateral tyre force dynamics using an extended Kalman filter from experimental road test data," Control Engineering Practice, vol. 17, no. 3, pp. 357-367, 2009.

[11] S. J. Julier and J. K. Uhlmann, "A new extension of the kalman filter to nonlinear systems," in Proceedings of the International Symposium on Aerospace/Defense Sensing, Simulation and Controls, pp. 182-193, Orlando, Fla, USA, April 1997.

[12] E. A. Wan and R. Van der Merwe, "The unscented Kalman filter for nonlinear estimation," in Proceedings of the IEEE Adaptive Systems for Signal Processing, Communications, and Control Symposium (AS-SPCC '00), pp. 153-158, IEEE, Lake Louise, Canada, October 2000.

[13] E. A. Wan and R. Van der Merwe, "The unscented Kalman filter," in Kalman Filtering and Neural Networks, chapter 7, Wiley, 2001.

[14] M. Doumiati, A. C. Victorino, A. Charara, and D. Lechner, "Estimation of vehicle lateral tire-road forces: a comparison between extended and unscented Kalman filtering," in Proceedings of the European Control Conference (ECC '09), pp. 48044809, Budapest, Hungary, August 2009.

[15] X. Shaobo and L. Cheng, "State and parameters estimation of vehicle based on UKF," Transactions of the Chinese Society of Agricultural Machinery, vol. 42, no. 12, pp. 6-12, 2011.

[16] W. Chu, S. Li, Q. Jiang, L. Liu, and Y. Luo, "Speed estimation for all-wheel drive vehicles based on multi-information fusion," Automotive Engineering, vol. 33, no. 11, pp. 962-996, 2011. 
[17] L. R. Ray, "Nonlinear tire force estimation and road friction identification: simulation and experiments," Automatica, vol. 33, no. 10, pp. 1819-1833, 1997.

[18] U. Kiencke and L. Nielsen, Automotive Control Systems, Springer, Berlin, Germany, 2000.

[19] H. B. Pacejka, Tire and Vehicle Dynamics, Elsevier, Oxford, UK, 2012.

[20] J. Dugoff, P. Fanches, and L. Segel, "An analysis of tire properties and their influence on vehicle dynamic performance," SAE Paper 700377, 1970.

[21] T. D. Gillespie, Fundamental of Vehicle Dynamics, SAE International, Warrendale, Pa, USA, 1992.

[22] R. Rajamani, Vehicle Dynamics and Control, Springer, Berlin, Germany, 2005.

[23] D. Guo and X. Wang, "Quasi-Monte Carlo filtering in nonlinear dynamic systems," IEEE Transactions on Signal Processing, vol. 54, no. 6, pp. 2087-2098, 2006.

[24] R. Van der Merwe and E. A. Wan, “The square-root unscented Kalman filter for state and parameter-estimation," in Proceedings of the IEEE Interntional Conference on Acoustics, Speech, and Signal Processing (ICASSP '01), pp. 3461-3464, Salt Lake City, Utah, USA, May 2001.

[25] H. Lee, "Reliability indexed sensor fusion and its application to vehicle velocity estimation," Journal of Dynamic Systems, Measurement and Control, Transactions of the ASME, vol. 128, no. 2, pp. 236-243, 2006.

[26] F. Yongling and Q. Xiaoye, LMS Imagine.Lab AMESim System Modeling and Simulation, Beihang University Press, Beijing, China, 2011. 


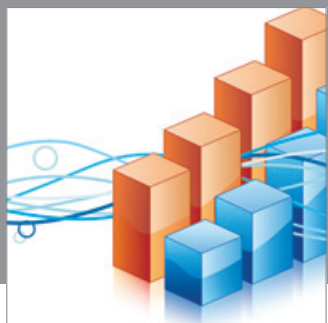

Advances in

Operations Research

mansans

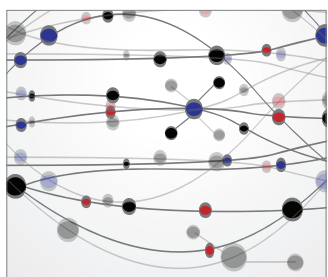

The Scientific World Journal
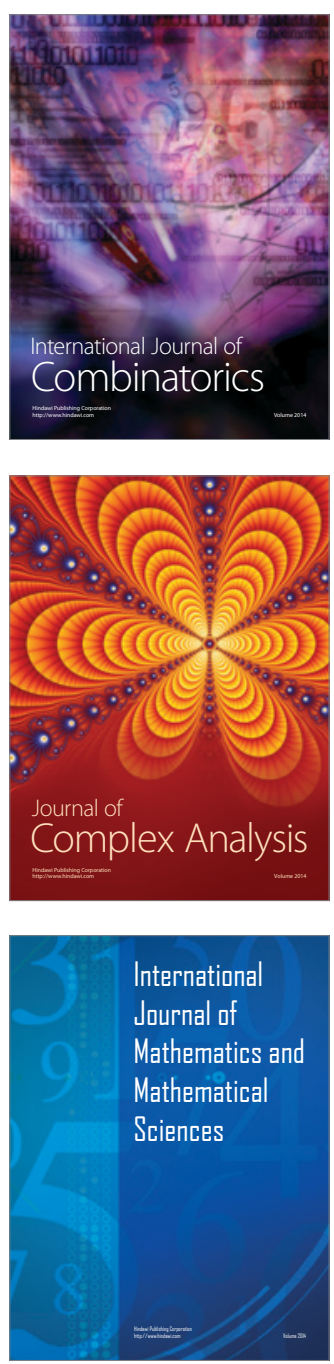
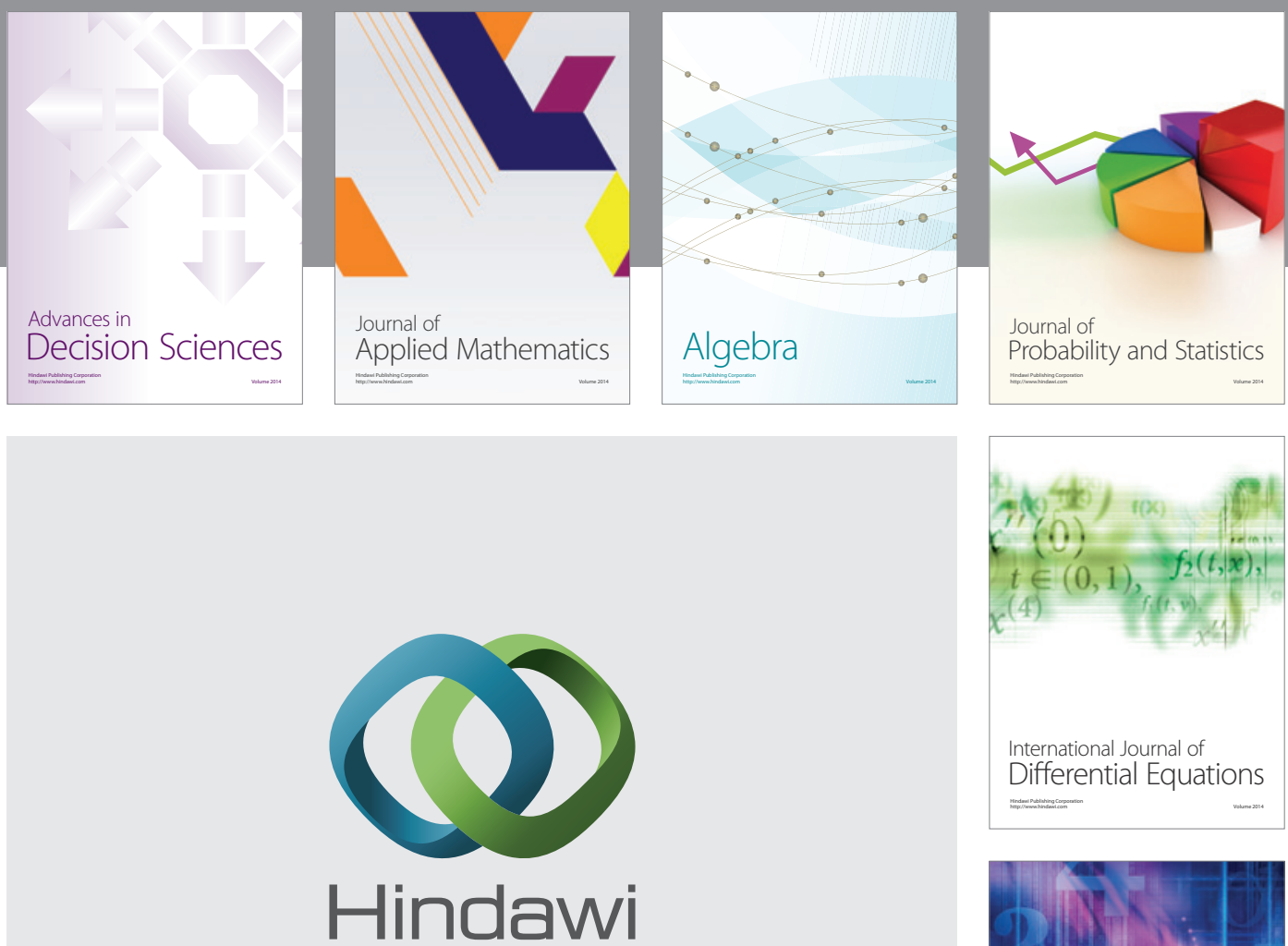

Submit your manuscripts at http://www.hindawi.com
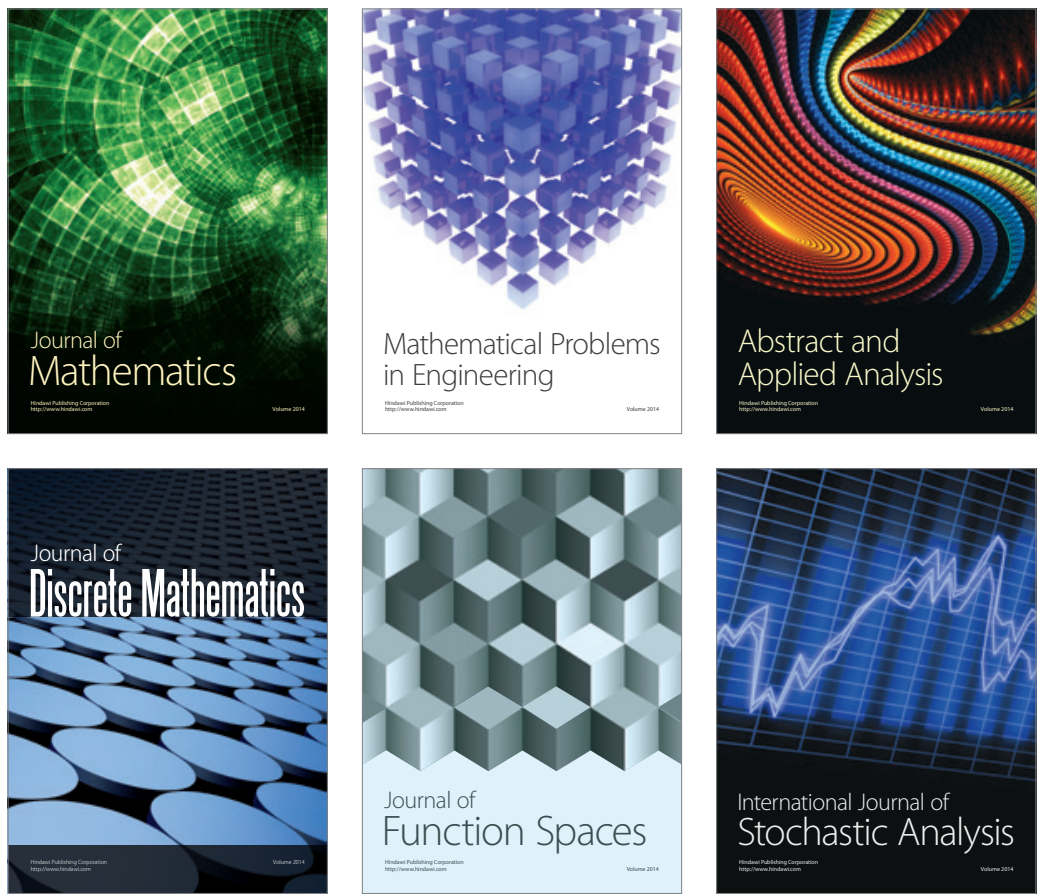

Journal of

Function Spaces

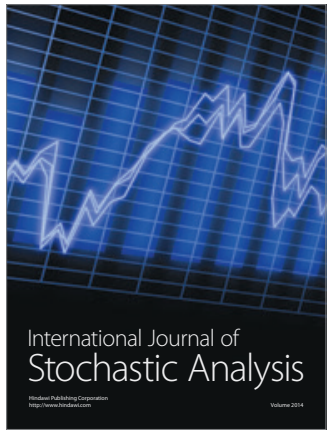

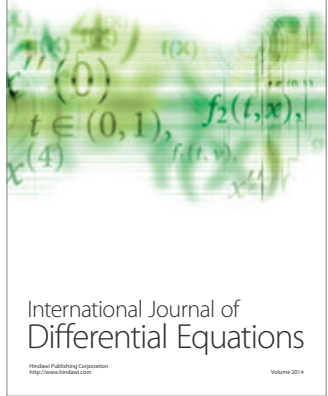
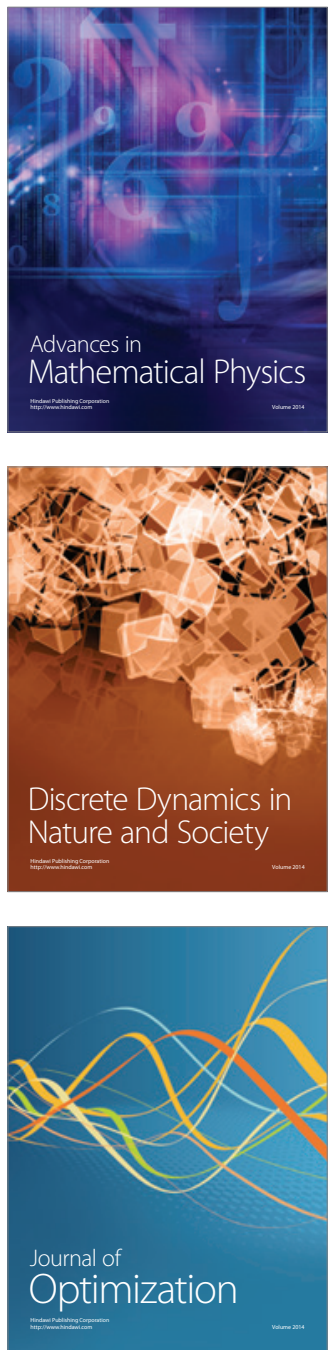